\title{
Evaluation of properties of AISI 431Grade Stainless Steel by Vacuum Annealing Process
}

\author{
Gandla Lakshmi Prasanna ${ }^{1 *}, G$. Keerthi Reddy ${ }^{1}$, and Dr. Ram Subbiah ${ }^{2}$ \\ ${ }^{1}$ PG Student, Mechanical department, Gokaraju Rangaraju Institute of Engineering and Technology, Hyderabad, India \\ ${ }^{2}$ Associate Professor, Mechanical Department, Gokaraju Rangaraju Institute of Engineering and Technology, Hyderabad, India.
}

\begin{abstract}
The wear behaviour of AISI 431 stainless steel was investigated at consistent load under a dry sliding touch with vacuum annealing device. The approach implemented was found to have been effective in improving the wear actions of stainless steel products, advancing the pliability properties. For Vacuum Annealed $\left(\mathrm{VA}_{1}\right) 2$ hours, Vacuum Annealed $\left(\mathrm{VA}_{2}\right) 4$ hours and Vacuum Annealed $\left(\mathrm{VA}_{3}\right) 6$ hours respectively, a low temperature heat treatment was performed on sample for the maximum annealing cycle. Untreated stainless steel (UNT) was used as a kind of viewpoint tool for contrast with specific hardening samples and conducted wear checks. The hardening samples show certain hard formed layers which enhance the hardness of the surface. Estimations of small scale toughness showed a crucial improvement in toughness following operation. This method raises the hardness of the substance up to 150 percent. A pin on the disc drive is used to do wear checking to assess wear failure. The layers were represented by optical lens magnification and the examination of electron magnifying glass.
\end{abstract}

\section{Introduction}

Much of stainless steel's research and creation seeks to create fresh concepts to enhance the mechanical properties. Development had become widespread in the 1980s of austenitic stainless steels with enhanced properties. Such products are commonly used for low temperature applications such as food processing equipment, nuclear plants, ocean management, producing petrochemicals, etc. However, rather bad wear habits seriously hamper the application of this substance on the face. To boost tribo-logical efficiency, many surface treatments are required but these are also of high cost nature. This paper addresses research undertaken on the effect of heat treatment for annealing on steel material [1]. The characteristics of the steel have been tested with concern in toughness and strength. Using optical microscopy, the samples were often studied microexamined. Hardness and longevity relied on the carbon content [2], the findings revealed. Thanks to heat treatment significant microstructural modification has also occurred. The aim of this research is to evaluate the mechanical properties of low carbon steel cold drawn which is subject to complete annealing [3]. The specimens of the Journal were slowly heated to a temperature of $900^{\circ} \mathrm{C}$, accompanied by a process of soaking in a muffle furnace of 60 minutes below that temperature [9]. Tensile, charpy and [4] Brinell toughness experiments were performed to assess the annealed steel's yield strengths, tensile strengths, impact strengths and resilience. The yield strength, tensile strength, toughness and resilience of the nails in the untreated nails was higher than that of

Completely rinsed nails at a soaking time of 60 minutes [8]. Examination of the microstructure showed that the rate of grain nucleation and recrystallization increased with a growing degree of cold-drawn deformation [5]. Grain production was observed at a higher degree of deformation due to the reduced mechanical properties of the material [11]. It is evident from the subsequent mechanical properties of the nails and the analysis of the microstructure that the necessary properties of the nails can be achieved by controlling the low carbon steel[12] character in the annealing phase. Mechanical properties such as the frequency of hardness and tension between three methods, the oil-quenching sample has the highest hardness and the annealed sample has the highest elongation. This is how heat treatment plays a key role in the mechanical properties of experimental steel and the resistance to corrosion [7].

\section{Experimental procedure}

The $150 \mathrm{~mm}$ diameter, $10 \mathrm{~mm}$ thick disk is hardened to its saturated maximum by a vacuum tempering operation. Pin content is $30 \mathrm{~mm}$ in length and $8 \mathrm{~mm}$ in diameter, respectively. The specimens are subject to phases of annealing. The specimens were machined and cut by electric discharge machine with wire cutting. The test method involves a laboratory technique for evaluating 
textile wear, using a pin-on-disk tool while slipping. In nominally non-abrasive conditions compounds are checked in pairs. The key areas of experimental interest are defined by utilizing this method of wear measurement apparatus. The friction coefficient is therefore calculated. Two specimens are used to perform the pin on disk wear test. The disk is rotated against a sliding pin which works with a constant charge. Untreated here with 6 conditioned samples shall be exchanged for wear check immediately. Wear tests for the pin and the disk separately are recorded as volume loss in millimetres [10]. The volume of wear is measured by calculating the correct dimensional measurements on both pre- and post-test specimens. In reality, dimensional wear scales are often used, as failure is too low to reliably quantify. If mass loss is calculated, the mass loss value is translated to volume loss using a reasonable formula for the density of the specimen. The following example demonstrates a schematic sketch of a standard pin-on-disk wear check. Here the mechanism consists of a powered spindle and chuck for holding the spinning disk, a lever-arm tool for keeping the pin and attachments for pressing the pin specimen with a controlled charge against the spinning disk specimen. The wear track on the disk is a loop, where multiple wear passes are obtained on the same road.

The composition of AISI431 Grade Stainless steel W.r.t A.Rajasekhar et al. i.e elements in percentage (\%) is carbon(c)- 0.17, Manganese (Mn)- 1, Silicon(Si)- 0.6, Phosphorus (P)- 0.04, Sulphur (S)- 0.03, Chromium (Cr)17, Nickel (Ni)- 2 and Ferrous (Fe)- 79.16

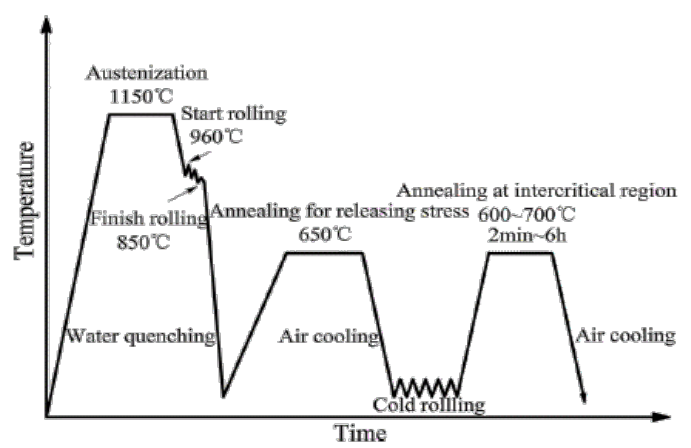

Fig 2.0. Schematic of Experimental Steel rolling and annealing process. W. r. t Jun Hul et al.

The wear test experimental results of samples and weight loss in grams (gms) are obtained as follows untreated sample (UNT) - 0.0066, Vacuum Annealed $1\left(\mathrm{VA}_{1}\right)$ $0.0039 \mathrm{gms}$, Vacuum Annealed $2\left(\mathrm{VA}_{2}\right)-0.0027 \mathrm{gms}$, Vacuum Annealed $3\left(\mathrm{VA}_{3}\right)-0.005$.
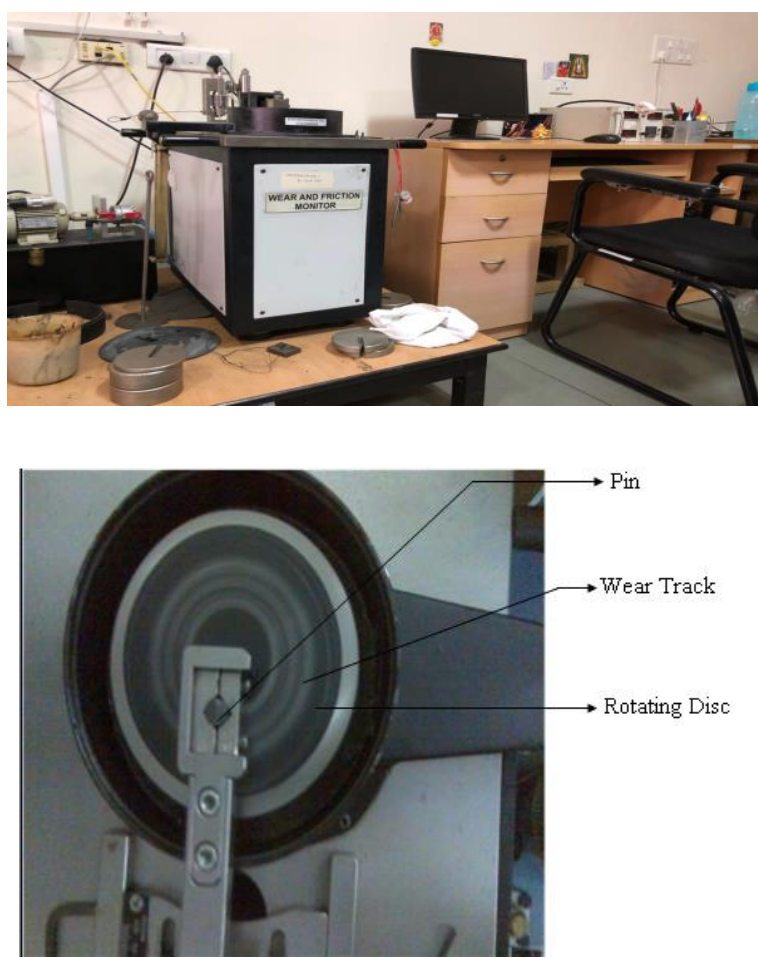

Fig. 2.1 Wear Track obtained on Wear Testing machine @ BITS, Hyderabad

\section{Results and Discussion}

The specimens were fitted for the microscopy test according to normal metallographic technique as per the ASTM requirements. The specimens were manually polished using emery papers accompanied by diamondpasted lap polishing [13]. The etching method was performed for 15 seconds in solution of oxalic acid at room temperature. Oxalic acid is used to expose the compound layer immediately on the immediate surface and in the case the solid nitrides. The specimens were promptly rinsed with water after etching, then vapour degreased to ensure consistent microstructure discovery. Clemex picture analyser was used to capture the microstructure of the heat treated specimens.

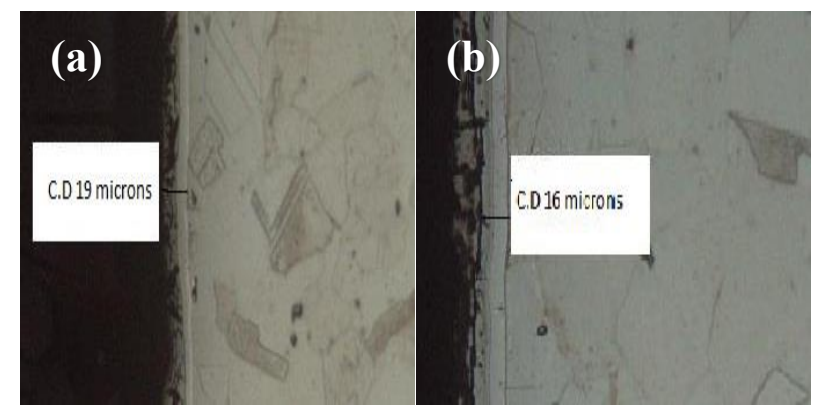




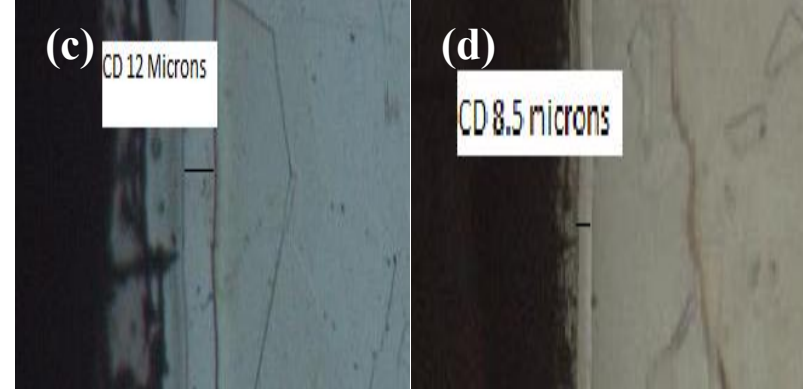

Fig 3.1 Microstructure and case depths of samples (a. Untreated specimen), (b. Vacuum Annealed 1(VA 1$)$ ), (C. Vacuum Annealed 2(VA2)), (d. Vacuum Annealed 3(VA3))

It has been observed from the aforementioned illustration that the untreated sample has a case depth of 19 microns while the samples subjected to the annealing phase have 16 microns, 12 microns and 8.5 microns respectively. Since the stainless steel content belongs to the division of martensitic, the depth of the case decreases throughout the process of annealing. Annealing process promotes ductility, softens the stainless steel material and hardness will be reduced. This should be noticed that the soil comprises a fairly uniform coating of ferrite and cement. The iron nitride formation may be due to the discontinuous white areas found at the top of the hardened base.

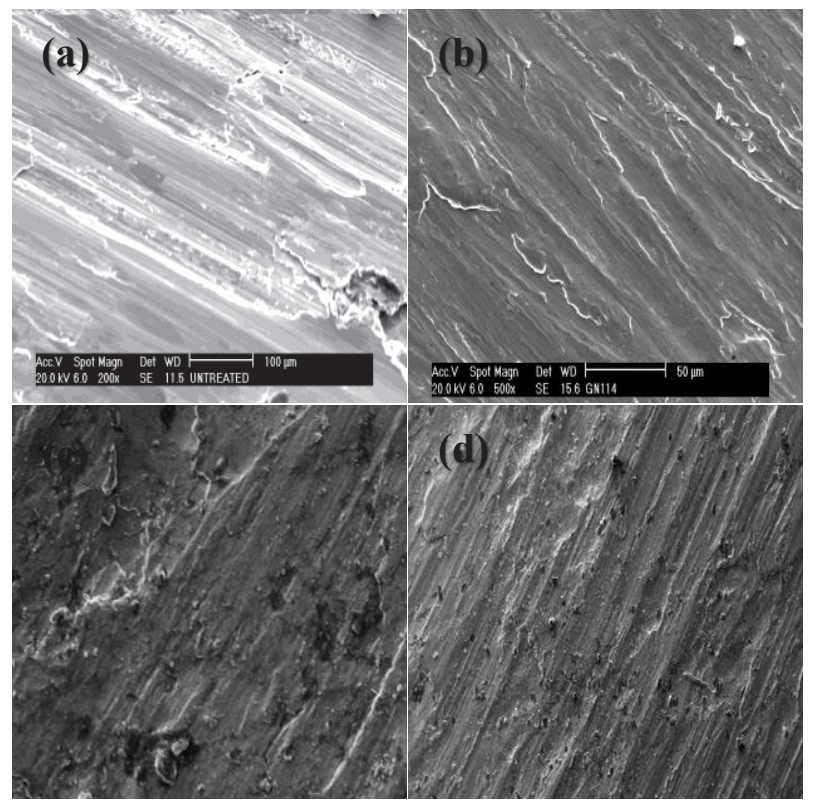

Fig 3.2 SEM images of (a. untreated specimen), (b. Vacuum annealed $\left.1\left(\mathrm{VA}_{1}\right)\right)$, (c. Vacuum annealed $\left.2\left(\mathrm{VA}_{2}\right)\right)$, (d. Vacuum annealed $\left.3\left(\mathrm{VA}_{3}\right)\right)$

\section{Conclusion}

The effect of the treatment method of vacuum annealing on the wear action of martensitic stainless steel AISI 431 was tested using a pin on the disk apparatus and eventually examined by different metallographic measurements. The main results taken from the research are:
1. The moderate oxidative wear mode was calculated within the spectrum of control variables used for wear monitoring. The key explanation for the wear rate decrease was attributed to the strengthening of martensitic matrix by fine carbide precipitates.

2. The martensitic fine grain structures were transformed into coarse grain structures during the annealing process. Through this way ductility and softness on the substrate is encouraged.

\section{References}

1. L.W.Tsay, Y.M.Chang, S.Torng, H.C.Wu, Journal of Materials Engineering and Performance, 114 (2002)

2. Y. Song, J. Cui, L. Rong, Journal of Materials Science \& Technology, 32 (2016)

3. S.K. Bonagani, V. Bathula, V. Kain, Corrosion Science, Volume 131, Pages 340-354.(2018).

4. A.Rajasekhar, G.Madhusudhan Reddy, T. Mohandas, V.S.R. Murti, Materials \& Design Volume 30, Issue 5, May 2009, Pages 1612-1624.

5. P. Wen, Z. Cai, Z. Feng, G. Wang, optics and laser technology, volume 75,Pages 207-231.(2015)

6. C.R. Anoop, A. Prakash, S.V.S. Narayana Murty, Indradev, Materials Science and Engineering, Volume 709, Pages 1-8.(2018).

7. T. Zhou, R.Prasath Babu, J. Odqvist, H. Yu, P. Hedstrom materials and design, volume 143, pages 141-149.(2018).

8. Tummala Suresh Kumar, Kosaraju Satyanarayana, Materials Today: Proceeding, 26 (2), 2020.

9. Manikonda, R. D., Kosaraju, S., Raj, K. A., \& Sateesh, N., Material Today 5, 20104-20109. (2018)

10. M.-S. Ding, J.-P. Du, L. Wan, S. Ogata, L. Tian, E. Ma, W.-Z. Han, J. Li , Z.-W. Shan, Nano Lett. 16 (2016) 4118-4124.

11. Rajagiri A, MN Sandhya, Nawaz S, Suresh Kumar T, E3S Web of Conferences 8701004 (2019)

12. T. Byun, E. Lee , J. Hunn , J. Nucl. Mater. 321 (2003) 29-39 .

13. Baloji, D., Anil, K., Satyanarayana, K., Singh, S. K., \& Naik, M. T. Material Today 18, 4475-4481. (2019) 